Session 2225

\title{
Teaching Electrical Engineering Design Using the Modified Decision Tree Approach
}

\author{
Domingo L. Uy \\ Fort Valley State University
}

\begin{abstract}
This paper presents the Modified Decision Tree Approach (MDTA) for teaching design in the context of Electrical Engineering. The salient feature of this method is that it facilitates the generation of alternative courses of actions, such that one can easily see the big picture, and at the same time putting the design in proper context. The method is illustrated by designing a simple one-stage transistor amplifier circuit. Using this method, one can easily obtain a very large number (in the thousands) of possible design structures or concepts. One can then "prune" the tree and select the "best" possible design structure. This method can also be applied to other fields of engineering.
\end{abstract}

\section{Introduction}

In recent years, teaching engineering design is getting more attention in engineering education. This is due to the ABET Engineering Criteria 2000 initiatives, which have changed the way instructors delivered their courses. In electrical engineering, ABET requires that all electrical engineering courses integrate design throughout the curriculum, and now there is also a need to demonstrate outcome assessment, and to check whether a program achieved its stated missions and objectives.

This paper presents a method for improving the way we teach engineering design. The name of this method is called the Modified Decision Tree Approach (MDTA). This method can provide a systematic framework for generating alternatives, by looking at a design problem in the broadest perspective possible and limited only by one's own knowledge and imagination. By using this method, one can see the big picture and the context of the design. The method can be very useful in showing how to design real-world problems.

This paper is organized as follows:

- Section 2 gives the overview of an engineering design process. This sets the stage for the introduction of the Modified Decision Tree Approach (MDTA);

- Section 3 gives a brief review of the Decision Tree Approach (DTA), and this helps 
introduce the MDTA in Section 4;

- Section 4 is about the MDTA and the advantages of using this method;

- Section 5 is about system modeling for the MDTA;

- Section 6 is about the "pruning" of the Modified Decision Tree (MDT), the discovery of "hidden" design criteria, and the selection of "best" design structure or concept;

- Section 7 gives an application of the MDTA in Electrical Engineering; and

- Section 8 gives the Conclusion.

\section{The Overview of An Engineering Design Process}

The Engineering Design process is an iterative process. It is a multi-dimensional problem in nature, and there are many factors to consider. It is also a form of problem solving technique ${ }^{4}$, and there is a need to generate alternative courses of actions. A flow-chart of an engineering design process is shown in Figure 1. In Figure 1, all the steps are pretty much in common for all branches of engineering, except for Block 5, which is discipline specific. And for all engineering disciplines, one can still find some similarities in their design techniques. In Block 8, an example of an engineering product is a hardware system. The types of engineering products are very broad and diversified. For examples, in electrical engineering, we have light bulbs, circuits, telephones motors, generators, transformers, calculators, personal computers, computer software, automatic data acquisition systems, etc.

The difficulty in teaching engineering design, is basically in Block 2, and this can become a "bottleneck" in the design process. It can take a lot of time to generate various alternatives. And when one finds an alternative, one normally follows a narrow path and finds out whether the idea works or not. If the idea works, the design continues to the next phase. But if it doesn't work, the process is repeated from the beginning. This process is pretty much like the trial and error approach.

The method presented in this paper will help alleviate this problem. The method will focus only on Blocks 2 , Block 3, and Block 4 of Figure 1 - the generation of alternative courses of actions, the selection of possible courses of actions, and the selection of the "best" possible course of action. In this paper, the "alternative courses of actions" is broadly interpreted. It can mean as: alternative system configurations, alternative system structures, alternative concepts, alternative chemical formulations, alternative algorithms, and etc.

\section{A Brief Review of the Decision Tree Approach (DTA)}

In this paper, a brief review of the Decision Tree Approach (DTA) is in order. This is useful in understanding the MDTA. The Decision Tree Approach is used in management decision models, 


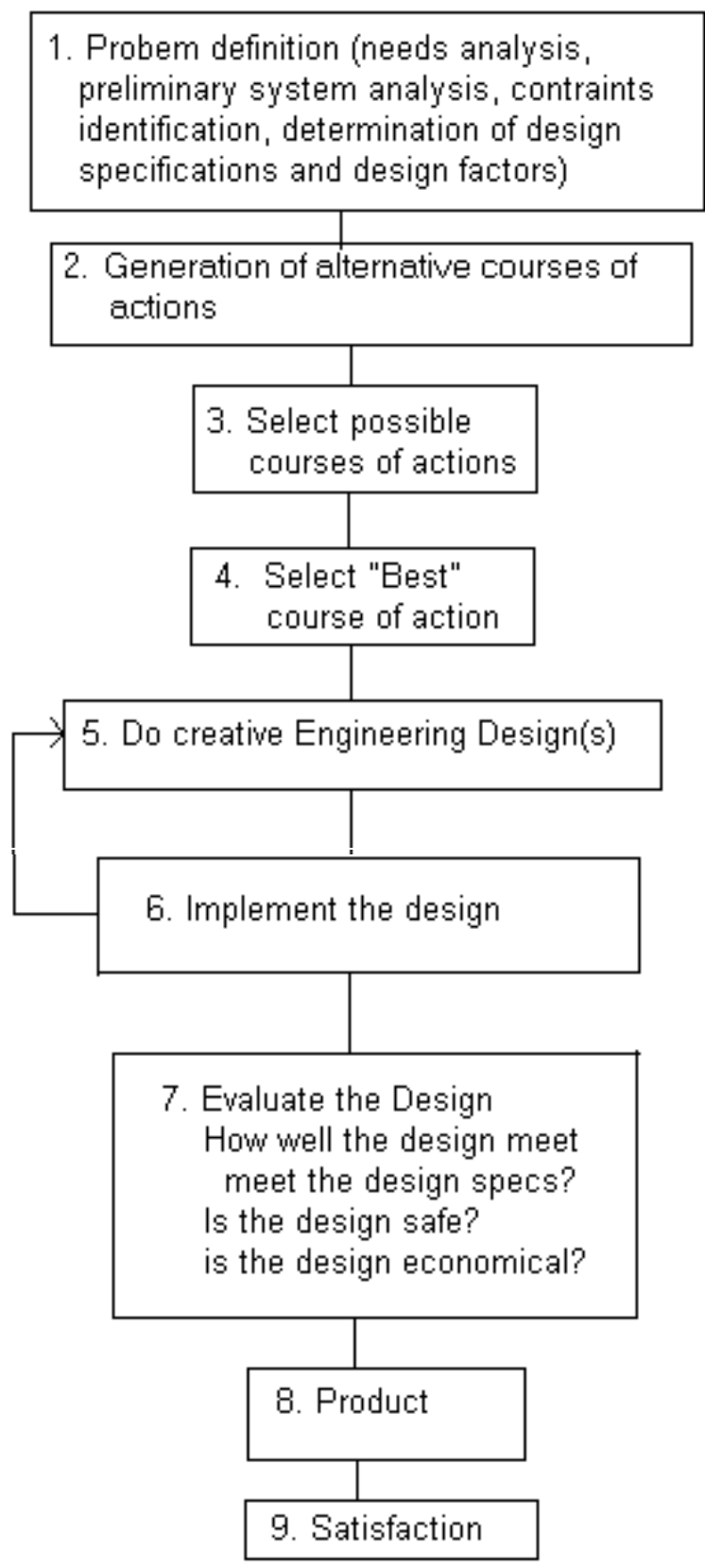

Figure 1. An Engineering Design Process 
in computer programming, and engineering design ${ }^{1,2,3}$. The Decision Tree Approach can be explained by considering the decision tree ${ }^{1}$ given in Figure 2. In this figure, the square node stands for the decision point (DP). Each decision point produces a certain number of alternatives, which are represented by branches of the tree. Each branch terminates in a circular node, which represents the state of nature, or the possible outcome of an alternative, which could occur. Each DP requires the decision maker to ask the question: How many ways can I accomplish this task? The possible answers to this question will yield the alternative courses of actions of this decision.

Figure 2 shows an example of a decision making process about investing ${ }^{1} \$ 1000.00$. When an individual invests in stock, the stock can go up or down, and the nature of this node (node 1) is as such, and the expected value (EV) of the node, or the alternative is $\$ 1280$, after taking into account the probabilities. Now, when one puts his/her money in the bank saving account (CD), the interest rate can go up or down, and the nature of this node (node 2) is as such. The expected value (EV) of this node, or this alternative is $\$ 1050$. In this case, using the "rollback" process ${ }^{1}$, the state of the node is evaluated right away. After the evaluation, the final decision is to invest in stock, because the $\mathrm{EV}$ of this node is higher.

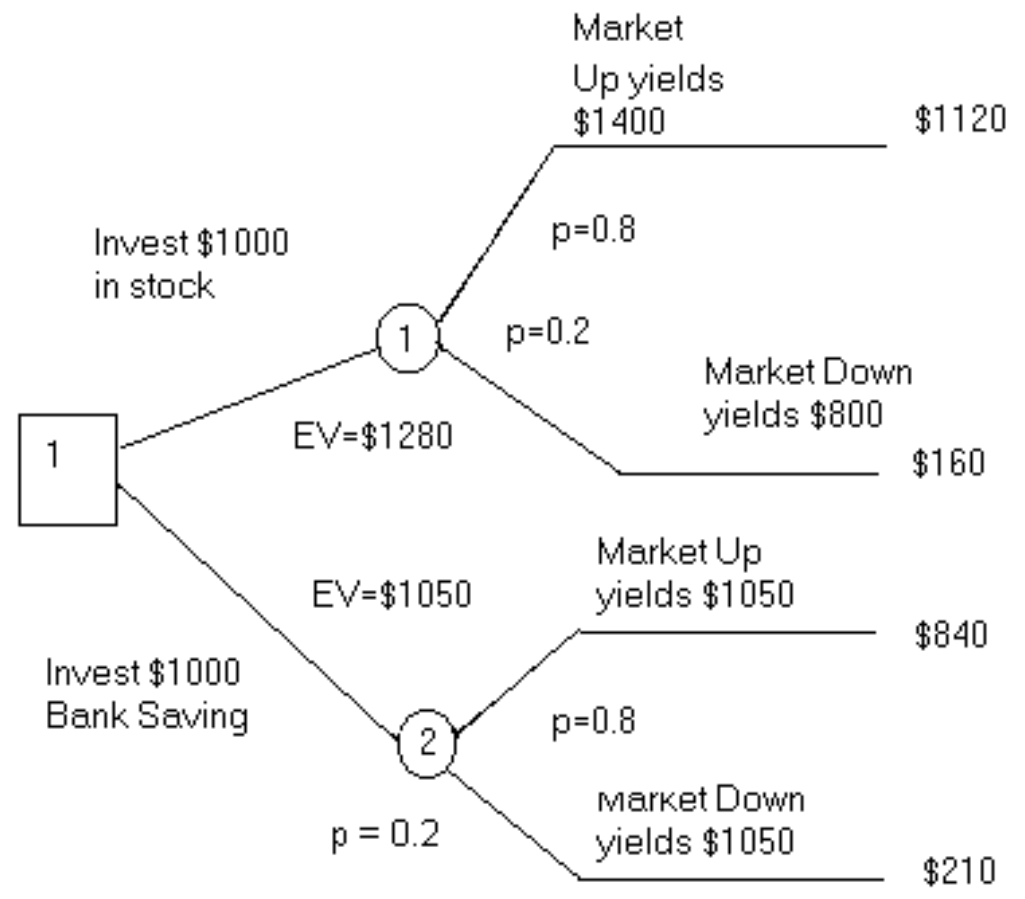

Figure 2. An example of DTA 


\section{The Modified Decision Tree Approach (MDTA)}

In the MDTA method, the tree (MDT) looks like the tree using the decision tree approach (DTA), but with some major modifications as described below:

1) The tree is constructed differently. It is constructed such that the outcomes of each decision point are deliberately postponed, until all the other decision points are included in the tree;

2) Each branch of a DP leads to another DP, and so on. It is the concatenation of decision points, until the "final" level of decision point is reached, after which the outcome or the state of nature of each node is evaluated. This is similar to building a whole system block by block, until the final block is in place. It is only after the whole system is constructed, that the evaluation process can then begin. But if one were to violate this rule, the system under consideration will be incomplete, and it will lead to possible going out of context and the outcomes may be undesirable;

3) Also, one can embed or built-in the design context within the MDT itself.

Note that for each decision point the branches of the tree are generated in the same way as in the DTA. The basic question to ask is: How many ways can I accomplish this task? An example of a three-level deep Modified Decision Tree (MDT) for the system S ( Figure 3) is shown in Figure 4. The square nodes are the decision points (DPs). The circular nodes are the outcomes. Each outcome consists of a set of alternatives. For example, in Figure 5, the outcome node $\mathrm{SS}_{2}$ contains the alternatives $\mathrm{m}, \mathrm{p}$, and $\mathrm{u}$. Also, in Figure 4, the outcomes $\mathrm{SSj}, \mathrm{j}=1,2, . .8$, can be interpreted as the alternative system design structures for the system S. In Figure 5, one can see that the system is defined by its system structure, and the system structure will in turn define the "hidden" design criteria for the system $\mathrm{S}$. This will be clarified in Section 7.

The advantages of using the MDTA are:

1) It allows the designer to see the big picture of the design alternatives;

2) It systematizes the generation of design alternatives. It enable the users approach design in an orderly fashion;

3) The design problem is general. This is in contrast to the conventional design method, where one normally wants to find a way to narrow down the scope of the design problem, right at the beginning. This step is taken in order to make the design problem more manageable. However, this approach may also eliminate many promising and unconventional design alternatives;

4) It can easily handle many other design factors including environmental, financial, and others. All one has to do is to augment a DP for each factor to an existing modified decision tree (MDT);

5) It requires the designer(s) to examine all possible outcomes or system structures, desirable and undesirable ones alike;

6) It communicates the decision making process to others in a very succinct manner;

7) It allows a design team to discuss the merit of each alternative system structure, one at a time;

8) It can serve as a good starting point for brain-storming session; 


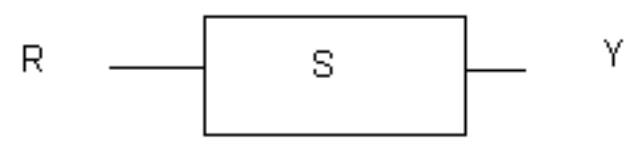

Figure 3. System $S$

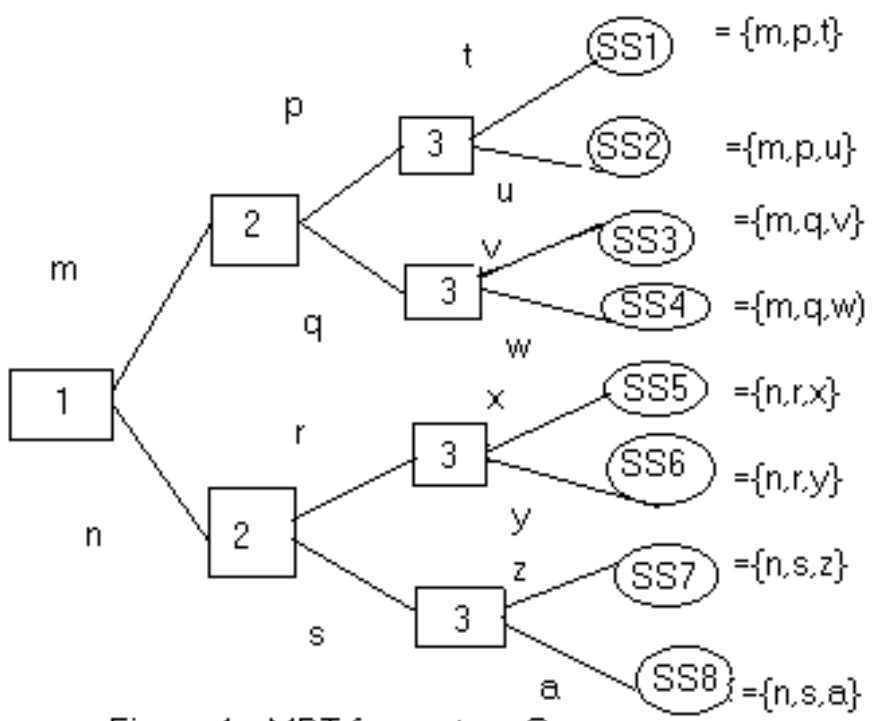

Figure 4. MDT for system S

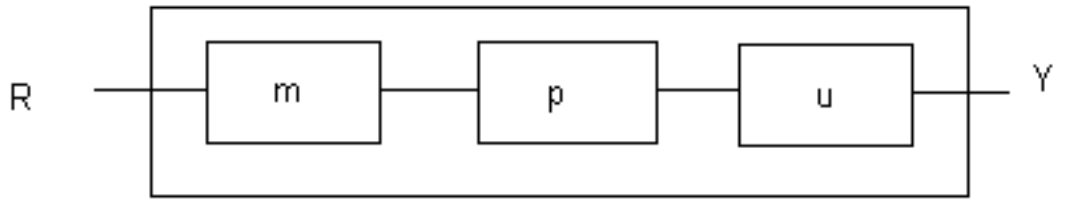

Figure 5. A design structure for system $S$

$$
\begin{aligned}
& \text { ญ్రి } \\
& \text { or } \\
& \text { ن্ } \\
& \text { के }
\end{aligned}
$$


9) It provides a way of unlocking the "hidden" design criteria, which are not "openly" specified at the beginning of a design. These "hidden" criteria are context-specifics in nature, and they are important in putting the design in proper context.

5. System Modeling for the MDTA

Assuming one is going to design a system S. In order to use the MDTA, one has to recast or model

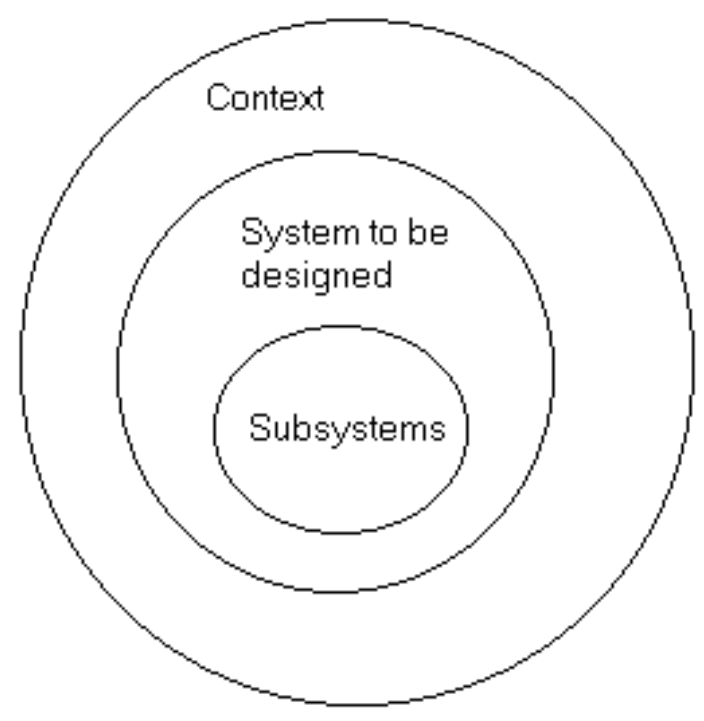

Figure 6

the design problem is a slightly different way. The guidelines are as follows:

a) One has to consider the context of the design problem. The context is the environment in which the system will operate. Since no real world design can exist in a vacuum, the design must be connected or link in some way to an environment, or to other systems and subsystems, and to the society as a whole. Design factors that are related to context are called the external factors of a design. For examples, we have safety, environmental, legal, political, economics, availability of resources, other systems in the neighborhood, just to name a few;

b) Also, one has to view the object to be designed as a system, and to identify the memberships of the system. The system memberships consist of subsystems, and a subsystem can have many sub-subsystems, and so on. In short, the system to be designed is broken down into subsystems, and each subsystem is further broken down into sub-subsystems, and so on, until 
one finally arrives at some "smaller" systems, which one can manageably design. A graphical representation of this relationship is shown in Figure 6. Design factors that are related to systems and subsystems are called the internal design factors. These factors are performance specifics. They will specify how a system or a subsystem will perform. For example, we have the voltage gain, bandwidth, rise-time, input impedance, and the stability of an amplifier;

c) Next, one has to construct a Modified Decision Tree (MDT), by asking questions that are system, subsystems, and context related. The following sample questions are helpful in generating the decision points (DPs) for the Modified Decision Tree:

How many ways can I design for environmental reason?

How many ways can I design for safety?

How many ways can I design for this subsystem?

How many ways can I design for this sub-subsystem?

How many ways can I accomplish this task?

and so on ...

The possible answers to these questions will form the branches of the tree. In short, at this stage of the design process, we are more interested in the conceptual development of the system design

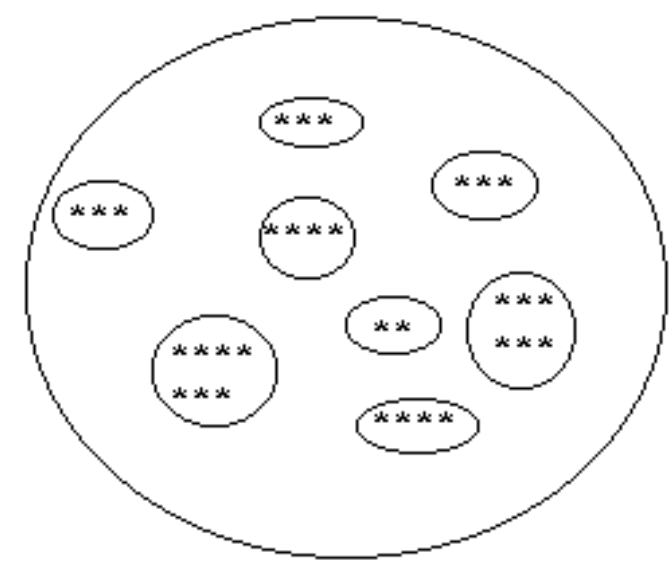

*-- One possible design $O-$ System structure

Figure 7. Relationship between system structure and single individual design

Structures, or the system concepts (Block 2 in Figure 1). Also at this point, we are not yet focusing our attention on how to get the system transfer functions, dynamic equations, or models. All of these will come later when we get into the details of the engineering design calculations, which is Block 5 in Figure 1.

To illustrate the MDTA, consider the system $\mathrm{S}$ as shown in Figure 3. The MDT is shown in Figure 4, 
which is a 3-level deep MDT. In order to obtain all the possible system design structures for the system $S$, let $n_{j}$ be the number of ways to implement the jth decision point, $D_{j}$, for $\mathrm{j}=1,2,3$. If $\mathrm{n}_{1}=2 ; \mathrm{n}_{2}=2$; and $\mathrm{n}_{3}=2$. Then, the total number of possible system structures, $\mathrm{n}_{\mathrm{T}}$, that one can use to design for the system $\mathrm{S}$ is given by

$$
\mathrm{n}_{\mathrm{T}}=(2)(2)(2)=8
$$

Figure 5 shows one possible way to realize the system S. Note that for each system structure or system concept, say $\mathrm{SS}_{2}$, one can still have many possible designs for that system. The relationship between a system structure and a particular system design is shown in Figure 7. In Figure 7, each star (*) in the system structure represents one possible design for the system $\mathrm{S}$. To

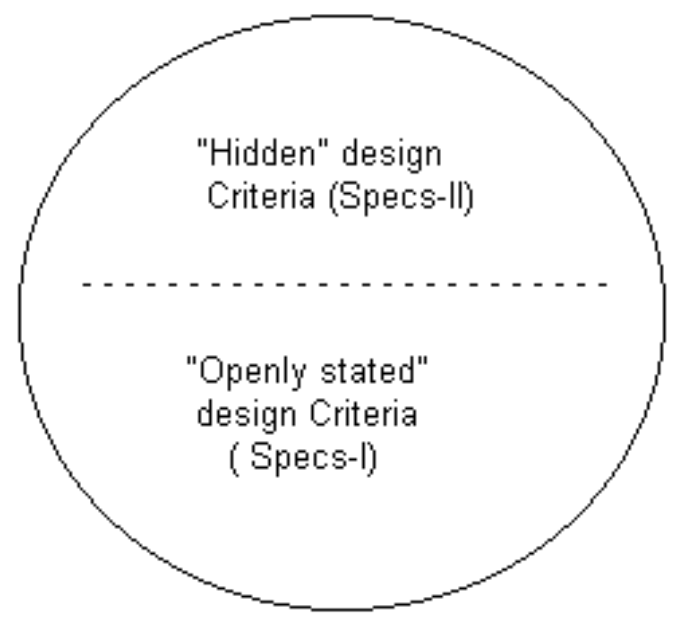

Figure 8. The overall or "complete" design criteria

clarify this point, if each system structure has an average of 200 possible designs, then in theory, there are $1600(8 \times 200)$ possible designs for the system $\mathrm{S}$. The general formula is:

Total no. of possible designs $=($ no. of system structures $) \times \quad$ (the average no. of system designs/system structure) 


\section{6. "Pruning" the MDT}

One can imagine that the size of the Modified Decision Tree (MDT) is very large. The whole tree for a given system, may have more than one thousand possible system structures. One can set out to draw this tree, but this is a very tedious and complicated process. So the next step is to find a way to simplify the tree and to obtain only the essential branches that have the possibility of satisfying the design specifications and constraints. This is called "pruning" the tree. "Pruning" the tree can be done in two stages.

\section{$\underline{\text { Stage } 1}$ Eliminate the Infeasible System Structures or Concepts}

The first stage is to prune the tree such that can one obtain all the feasible system structures or concepts that will satisfy the design constraints. And this is where one utilizes the design specifications, constraints, and one's own personal design experience in deciding which branches to cut. One should realize that it is possible during the construction of the MDT, incompatible subsystems were put together to synthesize the system. In this case, the designer has to check for incompatible subsystems, and to eliminate them during the pruning process.

After the pruning process, one can obtain a large number of feasible system structures. For example, consider again the MDT in Figure 4. A feasible design structure is constructed by tracing a path from level-1 to level-3 down to an outcome node. And along the way the system structure is constructed block by block, or subsystem by subsystem. An example is shown in Figure 5, where $\mathrm{SS}_{2}$ consists of the alternative courses of actions $\mathrm{m}, \mathrm{p}$, and $\mathrm{u}$.

The process of constructing one system design structure for the system S, whether it is the "best" one or not, will reveal a set of "hidden" design criteria, which are not obvious at the beginning of the design. In Figure 8 shows, the set of "hidden" criteria, let's call it Specs-II, complements the set of "openly stated" design criteria, let's call it Specs-I. The "hidden" criteria are context specifics, and the "openly stated" design criteria are performance specifics. Both sets of criteria are very important in designing real-world problems. The discovery of the "hidden" criteria will no doubt increase the number of overall design criteria, and these may complicate the design for the system $S$, but they will help qualify the context, which will make the design more effective and responsive.

\section{Stage 2. Select the "Best" Feasible System Structure or "Best" Concept for the Design}

In stage 1, once the pruning process is complete, we are still left with a large number of feasible system structures. Since a system (or product) cannot exist in a vacuum, one can introduce some realistic and relevant selection criteria, in selecting the "best" concept under the given circumstances. The following selection criteria can be introduced in the evaluation process. These are:

1) Physical realizability

2) Financial feasibility to create the system or the total cost of the system

3) Economic worthiness or profitability

4) Time factor

6) Availability of resources 
7) Properties of the feasible design structures or concepts in relation to performance, such as bandwidth, stability, speed of response, power output; and

8) Others

These criteria can become a checklist in the selection process. However, not all the criteria are relevant in a design. Thus, one has to pick the ones that are relevant to his or her own design situation.

A combination of subjective and objective selection criteria are used in picking the "best" concept in light of a given circumstances. Some of these criteria are difficult to quantify. It is a matter of estimation, intuition, subjective opinion, and trade-off, and it is up to the designer to use proper judgment.

If the total system cost is the only relevant factor, one can develop a cost function and then formulate the selection problem into a formal optimization problem $^{6}$. This is an advanced topic for undergraduate students in electrical engineering, and Optimization in itself is a graduate course in engineering and system sciences.

The process of selecting the "best" concept will also reveal a set of "hidden" selection criteria which may not be totally known at the beginning. This has to be expected, because this is the nature of engineering design process. As one advanced forward in the design process, a lot of unexpected problems will show up, and one must take it in stride and solve the problems as they come. This type of situation happens all the time in the real world. This is because one is creating a new system or product, that has never been done before, and it is hard to foresee all the aspects of the design right at the beginning. Note that the "hidden" selection criteria are also context-related, and these criteria together with the other "hidden" design criteria will constitute the overall "hidden" design criteria.

\section{Application of the MDTA to Electrical Engineering Design}

This example will show how to apply the MDTA in Electrical Engineering design. This example is about the design of a simple one-stage transistor amplifier. It will be shown that there are thousands of possible design structures for this system. The "best" system structure or concept is selected by establishing a set of realistic and relevant selection criteria.

\section{Design Problem:}

Design a one-stage transistor audio amplifier that will provide a gain of 50 (within 20\%) at midfrequency range for a load of $4 \mathrm{Kilo-Ohm}$ resistor. Also the low frequency cut-off is $600 \mathrm{HZ}$ (within 20\%), and the transistor input impedance is greater than $1000 \mathrm{Ohms}$.

A) Let us recast the problem into:

Design Problem : Design a one-stage transistor audio amplifier 
Design Performance Specifications:

$\begin{array}{ll}\text { Voltage gain, } & \left|\mathrm{A}_{\mathrm{V}}\right|=50, \text { within } 20 \% \\ \text { Load, } & \mathrm{R}_{\mathrm{L}}=4 \mathrm{~K} \\ \text { Input Impedance, } & \mathrm{Z}_{\mathrm{i}}>1000 \mathrm{Ohms} \\ \text { Low frequency Cut-off, } & \mathrm{f}_{1}=600 \mathrm{~Hz}, \text { within } 20 \%\end{array}$

B) Understand the problem:

To understand the problem, one must have some knowledge about the one-stage transistor amplifier system. One must also identify the members or parts of the amplifier, the other systems, and the environment in which the amplifier will operate. The results will be used to

obtain the proper context. An example is shown in Figure 9. Figure 9 shows the amplifier system to be designed, the subsystems, and the context of the design. The numerical number

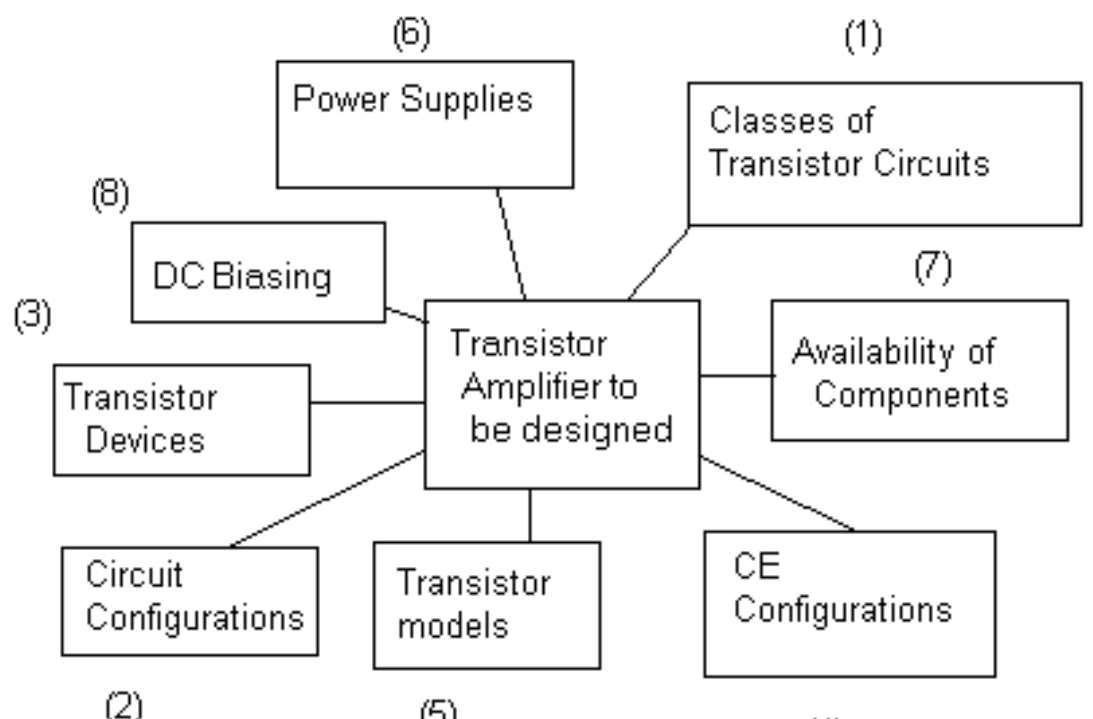

(2)

(5)

(4)

Figure 9. Putting the amplifier to be designed in proper context

associated with each block are used for referring the block, and for convenience. The number will also correspond to the question that will be generated later.

Again, referring to Figure 9, one can conduct a preliminary analysis as follows: A one-stage transistor amplifier circuit is compose of a transistor, resistors, and capacitors; There are many 
types of transistor devices (3); A transistor circuit requires a power supply (6) to operate; There are different classes of transistor amplifier circuits (1); There are also many ways that one can bias these circuits (8); There are different types of circuit configurations (2); There are also different types of CE configurations (4); There are different kinds of small-signal models that one can use (5) in the design; and also one has to consider the availability of the power supply system (6), and the circuit components (7).

Next, one will ask a series of questions about the system, the subsystems, and the context, and then provide answers to these questions. The basic question to ask is: How many ways can I accomplish this task?

C) Application of the MDTA

Let's apply the MDTA to this problem. From Figure 9, let's ask the following questions:

1) How many different classes of transistor circuits that one can use for this design? (System-related - DP1)

Possible answer $=3, \mathrm{n}_{1}=3$

BJT, JFET, MOSFET

2) How many kinds of circuit configurations that one can use for this design?

(System-related- DP2)

Possible answer $=9, \mathrm{n}_{2}=9$

for BJT: BJT Common-Base, BJT Common-Emitter, BJT Common-Collector

for JFET: JFET Common-Source, JFET Common-Gate, JFET Common-Drain

for MOSFET: MOSFET Common-Source, MOSFET Common-Gate, MOSFET

Common-Drain

3) How many types of transistor devices that one can used for this design?

(Subsystem-related - DP3)

Possible answer $=6, \mathrm{n}_{3}=6$

for BJT: PNP, NPN

for JFET: JFET n-channel, JFET p-channel

for MOSFET: MOSFET n-channel, MOSFET p-channel

4) How many kinds of Common-Emitter (CE) circuit configurations, that one can use for this design, in terms of the presence or absence of the bypassed capacitor, that is connected across the resistor $\mathrm{R}_{\mathrm{E}}$ ? (System-related - DP4)

Possible answer $=2, \mathrm{n}_{4}=2$

Common-Emitter with bypassed, Common-Emitter un-bypassed

5) How many kinds of transistor models that one can use for this design?

(Subsystem-related - DP5)

Possible answer $=3$ (or maybe more), $\mathrm{n}_{5}=3$

$\mathrm{r}_{\mathrm{e}}$-model, h-parameter model, $\pi$ - model 
6) How many levels of DC voltage for $\mathrm{V}_{\mathrm{CC}}$ (DC power supply) that one can use for this design? (Context- related - DP6)

Possible answer $=$ at least 3 (just to list a few), $\mathrm{n}_{6}=3$

$9 \mathrm{~V}, 12 \mathrm{~V}, 15 \mathrm{~V}$

7) How many kinds of transistors that are available commercially in the local area, that

one can use for this design, let say in Radio Shack? (Context-related - DP7)

Possible answer $=3$ (maybe more), $\mathrm{n}_{7}=3$

2N3906, 2N3904, 2N2222

8) How many types of DC biases that one can use for this design? (System-related- DP8)

Possible answer $=$ at least $4, \mathrm{n}_{8}=4$

Fixed-bias, Emitter-Stabilized bias, Voltage-Divider bias,

Collector-Feedback bias

Questions 1,2,4, and 8 are related to the system level; Questions 3 and 5 are related to the subsystem level; and Questions 6 and 7 are context-related questions. From these questions, one can apply the MDTA by constructing an 8-level deep modified decision tree (MDT). If one will trace all the possible branches from level-1 to level-8, one can obtain the total number of possible system (circuit) configurations, $\mathrm{n}_{\mathrm{T}}$, which is equal to

$$
\begin{aligned}
& \mathrm{n}_{\mathrm{T}}=\prod_{\mathrm{j}=1 \text { to } 8} \mathrm{n}_{\mathrm{j}}=(3)(9)(6)(2)(3)(3)(3)(4)=34,992 .
\end{aligned}
$$

A very small portion of the modified decision tree (MDT) is shown in Figure 10.

D) "Pruning" the MDT

Stage 1: Eliminate the Infeasible System Structures or Concepts

The MDT in Figure 10 is an 8-level deep, and one can imagine that the size of the whole tree is complicated, and not all system structures will be able to meet the design performance criteria (Specs-I). Let's apply the design specifications and constraints, and one's own design experience in "pruning" the tree. A good starting point is to look at the list of questions generated in part (C), and evaluate the answers.

1) In Question 1, one can rule out the classes of JFET and MOSFET circuits, since these 


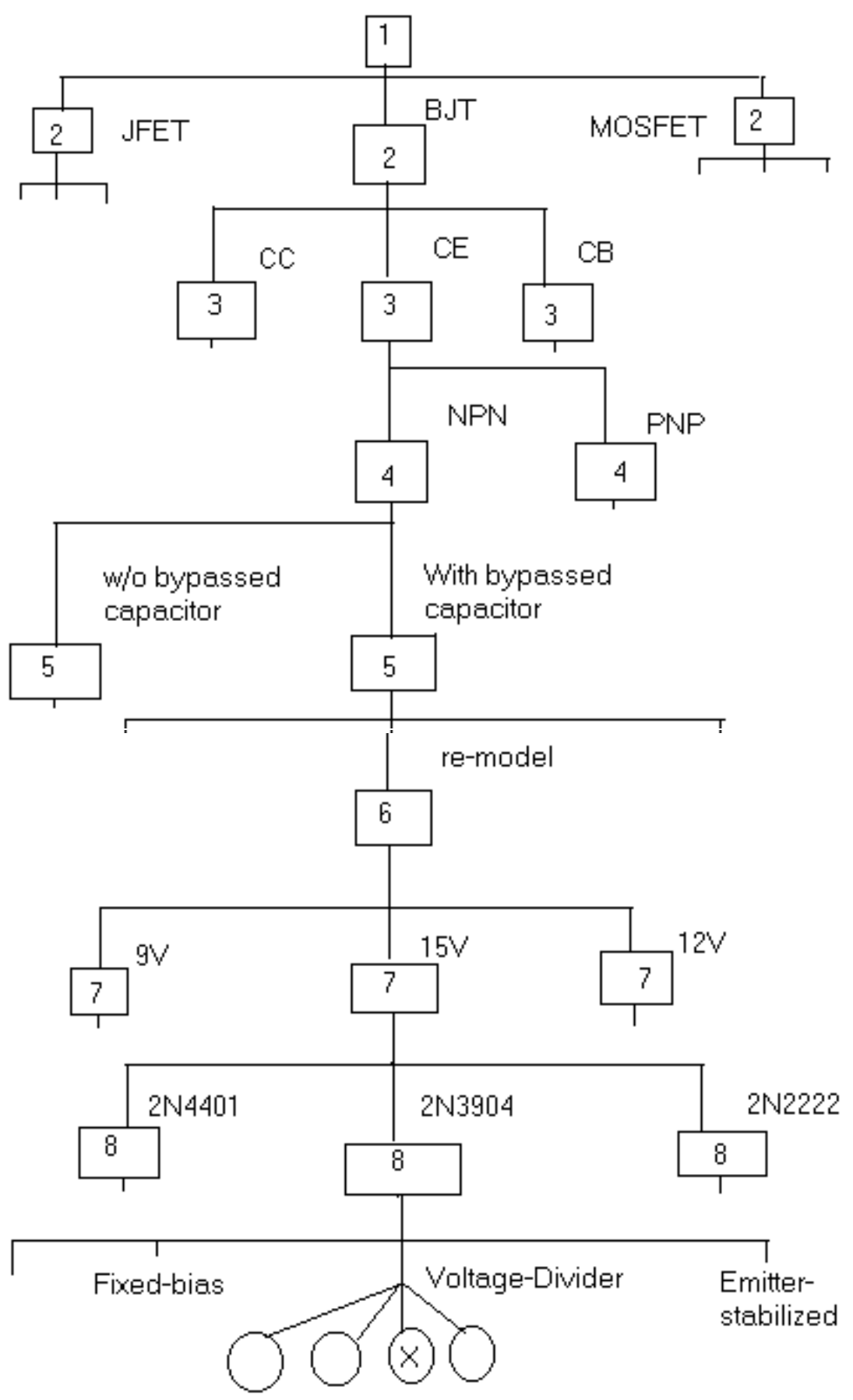

Figure 10. The MDT for the amplifer system

$$
\begin{aligned}
& \text { ญ̊ } \\
& \text { or } \\
& \text { ஸ্ } \\
& \vec{v}
\end{aligned}
$$


types of circuits can only yield low voltage gains, which is around 2 to 15 . Thus, a gain of 50 will require more than one stages of amplifiers, and this is not economical. So we can cut these branches out from the tree. Set $n_{1}{ }^{\prime}=1$.

2) In Question 2, the BJT Common-Base circuit configuration is out, since the input impedance of this amplifier is very low, which is around $20 \mathrm{ohms}$ or less, and the design specification for the input impedance, $Z_{i}$ cannot be met. So we can cut this branch out. We

can also rule out the Common-Collector configuration ( Emitter-Follower Circuit), because the circuit configuration can only yield at most a gain of 1 . Thus, the voltage gain specification cannot be met. So, we can also cut this branch out. The Common-Emitter configuration can do the job, so we keep this branch of the tree. Set $\mathrm{n}_{2}^{\prime}=1$.

3) In Question 3, both types of transistors can do the job, so we keep both branches. Set $\mathrm{n}_{3}{ }^{\prime}=2$.

4) In Question 4, we can rule out the un-bypassed CE circuit configuration, since this can yield only low voltage gain (usually less than 5). So we can cut this branch out. The bypassed CE configuration can do the job, so we keep this branch. Set $\mathrm{n}_{4}{ }^{\prime}=1$.

5) In Question 5, all the branches are possible, and we keep all the branches. Set $n_{5}{ }^{\prime}=3$.

6) In Question 6, we keep all the branches, but this will depend on the availability of the power supply. Set $\mathrm{n}_{6}{ }^{\prime}=3$.

7) In Question 7, all three can do the job, so we can keep all the branches. Set $n_{7}{ }^{\prime}=3$.

8) In Question 8, we have to rule out the fixed-bias configuration, since the bias-point of this circuit is not very stable, and we can keep the rest of the branches. Set $n_{8}{ }^{\prime}=3$.

Thus, after this round of pruning, we can find the total number of possible system design structures or configurations, $n_{T}$ '. This value is equal to the product of $n_{j}$, for $j=1,2 \ldots 8$.

$$
\mathrm{n}_{\mathrm{T}}^{\prime}=(1)(1)(2)(1)(3)(3)(3)(3)=162
$$

Thus, we have a total of 162 feasible system design configurations, for the amplifier. From Figure 10 , we can construct one feasible system design structure by tracing a path from the branches of level-1 to level-8 down to an outcome node. Note that Figure 10 follows closely, in proper order, the eight Decision Points (DPs) given in this example, that is, DP1 corresponds to Question 1; DP2 corresponds to Question 2; and so on.

\section{Stage 2. Select the "Best" Design Structure or Concept}

From Stage 1, we have 162 possible design concepts or design structures, that will meet the design specifications and constraints. Applying the first three selection criteria in Stage 2 of Section 6, we have the following: i) By inspection, all the feasible design concepts are physically realizable; ii) The financial feasibility criteria is not a problem, since this system is very inexpensive to build, and the cost (less than $\$ 10.00$ for a complete system) is not a problem; iii) The economic profitability criteria will not play a role in this case, since we are neither going to sell nor mass produce the amplifier. So one has to look for other subjective and objective selection criteria in the evaluation process, and one can consider the following for this design. These are:

a) The transistor model and the time to complete the design. This item is related to 


\section{Question 5 in stage 1;}

b) The availability of DC power supply. This item is related to Question 6 ;

c) The availability of transistors in the lab. This item is related to Questions 3 and 7; and d)

The DC biases. This item is related to Question 8.

\section{Evaluation Process:}

a) The transistor model and the time needed to complete the design. The selection of the transistor's small signal model and the time it takes to complete the design calculation are closely related. In this case, we want to be able to complete the circuit design in a short amount of time.

The use of the $r_{e}$-model is preferred over the other two models (see Section 7), because the model is relatively simple to use, and a complete paper design can be done in 10 to 15 minutes. Note that the transistor is a nonlinear device, and the parameters are not fixed, and they are also temperature dependent. Thus, there is no point of using a complicated model in this particular design, especially when the accuracy of the transistor parameters are not available. The best that one can hope for is to use the typical values of parameters from the manufacturer data sheets.

Thus, it can take a lot more time to design a transistor circuit using the other two models.

The $\mathrm{r}_{\mathrm{e}}$-model makes the design simple and straight forward. Although the voltage gain accuracy is around $20 \%$ to $30 \%$ compared to the actual circuit voltage gain. From experience, time is better spent in the laboratory for re-designing and tweaking the values of the resistors, such that the design performance specifications are met.

b) The availability of power supply. We have a variable DC power supply in the Lab, and the selection of $15 \mathrm{~V}$ for $\mathrm{V}_{\mathrm{CC}}$ is just a matter of choice. The other reason is to place the $\mathrm{DC}$ bias point close to the center of the linear region of the transistor characteristics. This will allow a larger input signal swing, say up to $100 \mathrm{mV}$ peak-peak, without hitting the saturation and cut-off regions.

c) The availability of transistors in the lab. We have some 2N2222, 2N3906, and 2N3904 transistors in the lab. The selection of $2 \mathrm{~N} 3904$ is a matter of preference. The type of transistor to use follows directly from 2N3904, which is an NPN transistor.

d) The DC biases. We have to consider the properties of each type of DC biasing. There are 3 possibilities: (a) Emitter-Stabilized bias configuration, (b) Voltage-Divider bias configuration, and (c) Collector Feedback bias configuration. Let's evaluate each circuit configuration, with respect to the following properties:

1) DC-Bias Stability with respect to the change in $\beta$ :

(c) is the most stable, followed by (b), and then (a).

2) Ease of Design calculations:

(a) is the easiest, followed by (b), and then (c).

3) Voltage-Gain Yield:

(a) and (b) are fairly high, and (c) is lower due to the effect of feedback.

Thus, it is clear from (3) that we have to choose either (a) or (b). From (1) and (2), there is a trade- 
off between ease of design and bias stability. Since bias stability is more important, then (b) is the logical choice. Thus, the Voltage-Divider bias configuration is selected.

To summarize the results, the selection criteria for picking the "best" concept from the list of 162 possible concepts are:
a) Use $r_{\mathrm{e}}$-model for the transistor
b) Use $\mathrm{V}_{\mathrm{CC}}=15 \mathrm{~V}$
c) Use an NPN transistor
d) Use 2N3904 transistor
e) Use the Voltage-Divider DC biasing

All the above items will form part of the overall "hidden" design criteria ( Spec-II). Thus, the overall "hidden" design criteria (Specs-II) or the context-related design specifications are:
1) BJT transistor
(Question 1)
2) $\mathrm{CE}$ configuration
(Question 2)
3) NPN transistor
(Question 3)
4) $\mathrm{CE}$ with Emitter-bypassed capacitor
(Question 4)
5) $r_{\mathrm{e}}$-model for the transistor
(Question 5)
6) $\mathrm{V}_{\mathrm{CC}}=15 \mathrm{~V}$ power supply
(Question 6)
7) 2 N3904 general purpose transistor (Question 7)
8) Voltage-Divider DC bias configuration (Question 8)

In Figure 10, the "best" design structure or concept can be obtained from the MDT by tracing the path from DP1 down to DP8, and to the outcome node X.

E) Do Creative Engineering Design (This step corresponds to Block 5 in Figure 1.)

The Overall Design Criteria:

(i) Use the Overall "Hidden" Design Specifications (Specs-II) and

(ii) Use the Design Performance Specifications (Specs-I):

$$
\begin{aligned}
& |A V|=50, \text { within } 20 \% \\
& R_{L}=4 K \\
& Z_{i}>1000-O h m \\
& f_{1}=600 \mathrm{~Hz}, \text { within } 20 \%
\end{aligned}
$$

(iii) Specify the Subsystem Level Design Specifications:

For the Transistor DC-Bias: Pick $\mathrm{I}_{\mathrm{CQ}}=3 \mathrm{~mA} ; \mathrm{V}_{\mathrm{CEQ}}=7.5 \mathrm{~V}$

Design Calculations:

Design working equations: 


$$
\begin{aligned}
& \mathrm{A}_{v}=-\left(\mathrm{R}_{\mathrm{c}} \| \mathrm{R}_{\mathrm{L}}\right) / \mathrm{r}_{\mathrm{e}} \\
& \mathrm{r}_{\mathrm{e}}=26(\mathrm{mV}) / \mathrm{I}_{\mathrm{E}}(\mathrm{mA})
\end{aligned}
$$

Transistor: $2 \mathrm{~N} 3904, \beta=160$

1) Let $I_{E}=I_{C}$; then $r_{e}=26 / 3=8.667$ Ohms.

2) $|A v|=\left|R_{C} \| R_{L}\right| r_{e}=50$; it yields $R_{C}=486$ Ohms.

Use $R_{C}=470$ Ohms (Standard (STD))

3) KVL equation: $V_{C C}-V_{C E Q}-I_{C} R_{C}-I_{E} R_{E}=0$. Use $V_{C C}=15 V ; I_{C}=I_{E}$

$$
\begin{aligned}
& 15-7.5-3(0.470)=3 R_{E} \\
& R_{E}=2.03 K . \text { Use } R_{E}=2.0 K(S T D)
\end{aligned}
$$

4) $\mathrm{V}_{\mathrm{E}}=\mathrm{I}_{\mathrm{E}} \mathrm{R}_{\mathrm{E}}=3(2.03)=6.09 \mathrm{~V}$

5) $\mathrm{V}_{\mathrm{B}}=\mathrm{V}_{\mathrm{E}}+\mathrm{V}_{\mathrm{BE}}=6.09+0.7=6.79 \mathrm{~V}$

6) Use: $V_{B}=V_{C C}\left(R_{2} /\left(R_{1}+R_{2}\right)\right)$; Assume $\beta R_{E}<=10 R_{2}$

$$
\mathrm{R}_{1}=1.209 \mathrm{R}_{2}, \mathrm{R}_{2}<=32.48 \mathrm{k}
$$

Pick $R_{2}=22 \mathrm{~K}(\mathrm{STD})$, then $\mathrm{R}_{1}=26.59 \mathrm{~K}$

Use $\mathrm{R}_{1}=27 \mathrm{~K}$ (STD)

7) Calculate $Z_{i}=R_{1}\left\|R_{2}\right\| \beta r_{e} ; \quad Z_{i}=1.24 K>1 K$

8) For the low-frequency cut-off ${ }^{5}, f_{1}$, the working equations are:

$$
\begin{aligned}
& \mathrm{f}_{\mathrm{LC1}}=1 /\left(2 \pi\left(\mathrm{R}_{\mathrm{S}}+\mathrm{Z}_{\mathrm{i}}\right) \mathrm{C}_{1}\right) \\
& \mathrm{f}_{\mathrm{LC} 2}=1 /\left(2 \pi\left(\mathrm{R}_{\mathrm{L}}+\mathrm{R}_{\mathrm{C}}\right) \mathrm{C}_{2}\right) \\
& \mathrm{f}_{\mathrm{LE}}=1 /\left(2 \pi \mathrm{R}_{\mathrm{e}} \mathrm{C}_{\mathrm{E}}\right) \\
& \text { where } \mathrm{R}_{\mathrm{e}}=\mathrm{R}_{\mathrm{E}}\left\|\left(\left(\mathrm{R}_{\mathrm{s}^{\prime}} / \beta+\mathrm{r}_{\mathrm{e}}\right)\right) ; \quad \mathrm{R}_{\mathrm{s}^{\prime}}=\mathrm{R}_{\mathrm{s}}\right\| \mathrm{R}_{1} \| \mathrm{R}_{2}
\end{aligned}
$$

$R_{\mathrm{s}}$ is the source generator resistance. In here, we assumed $R_{\mathrm{s}}=100-\mathrm{Ohm}$. We set: $f_{L C 1}=200 \mathrm{~Hz} ; f_{L C 2}=300 \mathrm{~Hz}$; and $f_{L E}=600 \mathrm{~Hz}$. $f_{L E}$ in general dictates the value for $f_{1}$. Using the formulas above, we get

$$
\begin{array}{lll}
\mathrm{C}_{1}=0.59 \mathrm{uF} \text {, use } 0.47 \mathrm{uF} & \text { (STD) } \\
\mathrm{C}_{2}=0.118 \mathrm{uF} \text {, use 0.1 uF } & \text { (STD) } \\
\mathrm{C}_{\mathrm{E}}=28.67 \mathrm{uF} \text {, use 33 uF } & \text { (STD) }
\end{array}
$$

F) Implement the Design (Block 6 in Figure 1)

The design values are given in Table 1 (Trial \#2). The final design circuit is shown in Figure 11. 
Table 1. Hardware Implementation

\begin{tabular}{lllll}
\hline \multicolumn{3}{c}{ Design Value } & \multicolumn{2}{c}{ Actual Value Used } \\
\hline Component (Calculated) & Standard & Trial \#1 & Trial \#2 \\
\hline $\mathrm{R}_{\mathrm{C}} \quad 0.486 \mathrm{~K}$ & $0.47 \mathrm{~K}$ & $0.56 \mathrm{~K}$ & $0.56 \mathrm{~K}$ \\
$\mathrm{R}_{\mathrm{E}} \quad 2.03 \mathrm{~K}$ & $2.0 \mathrm{~K}$ & $2.0 \mathrm{~K}$ & $1.69 \mathrm{~K}$ \\
$\mathrm{R}_{1} \quad 27 \mathrm{~K}$ & $27 \mathrm{~K}$ & $27 \mathrm{~K}$ & $27 \mathrm{~K}$ \\
$\mathrm{R}_{2} \quad 22 \mathrm{~K}$ & $22 \mathrm{~K}$ & $22 \mathrm{~K}$ & $22 \mathrm{~K}$ \\
$\mathrm{C}_{1} \quad 0.59 \mathrm{uF}$ & $0.47 \mathrm{uF}$ & $0.47 \mathrm{uF}$ & $0.47 \mathrm{uF}$ \\
$\mathrm{C}_{2} \quad 0.118 \mathrm{uF}$ & $0.1 \mathrm{uF}$ & $0.1 \mathrm{uF}$ & $0.1 \mathrm{uF}$ \\
$\mathrm{C}_{\mathrm{E}} \quad 34.41 \mathrm{uF}$ & $33 \mathrm{uF}$ & $33 \mathrm{uF}$ & $33 \mathrm{uF}$ \\
& & & & \\
\hline
\end{tabular}

Table 2. Hardware Test Results and Evaluation

\begin{tabular}{lllc}
\hline Item & Design Specs & Trial \#1 & Trial \#2 \\
\hline $\mathrm{V}_{\mathrm{CE}}(\mathrm{V})$ & 7.5 & 8.5 & 8.3 \\
$\mathrm{I}_{\mathrm{C}}(\mathrm{mA})$ & 3.0 & 2.5 & 3.01 \\
\hline$\%$ diff $\mathrm{V}_{\mathrm{CE}}$ & ----- & $13.33 \%$ & $10.66 \%$ \\
$\%$ diff $\mathrm{I}_{\mathrm{C}}$ & ----- & $16.66 \%$ & $0.33 \%$ \\
\hline$\left|\mathrm{A}_{\mathrm{V}}\right|$ & 50 & 34.37 & 45 \\
$\mathrm{Z}_{\mathrm{i}}$ & $1.24 \mathrm{~K}$ & $3 \mathrm{~K}$ & $2 \mathrm{~K}$ \\
$\mathrm{f}_{1}(\mathrm{HZ})$ & 600 & 488 & 606 \\
\hline$\% \operatorname{diff}\left|\mathrm{A}_{\mathrm{V}}\right|$ & ----- & $31.26 \%$ & $10 \%$ \\
$\%$ diff $\mathrm{f}_{1}$ & ---- & $18.67 \%$ & $1 \%$ \\
\hline
\end{tabular}

G) Evaluate the Design (Block 7 in Figure 1)

This example shows a design of a one-stage transistor amplifier system. The design met all the design specifications, and the final circuit is shown in Figure 11. The MDT for this example is an 


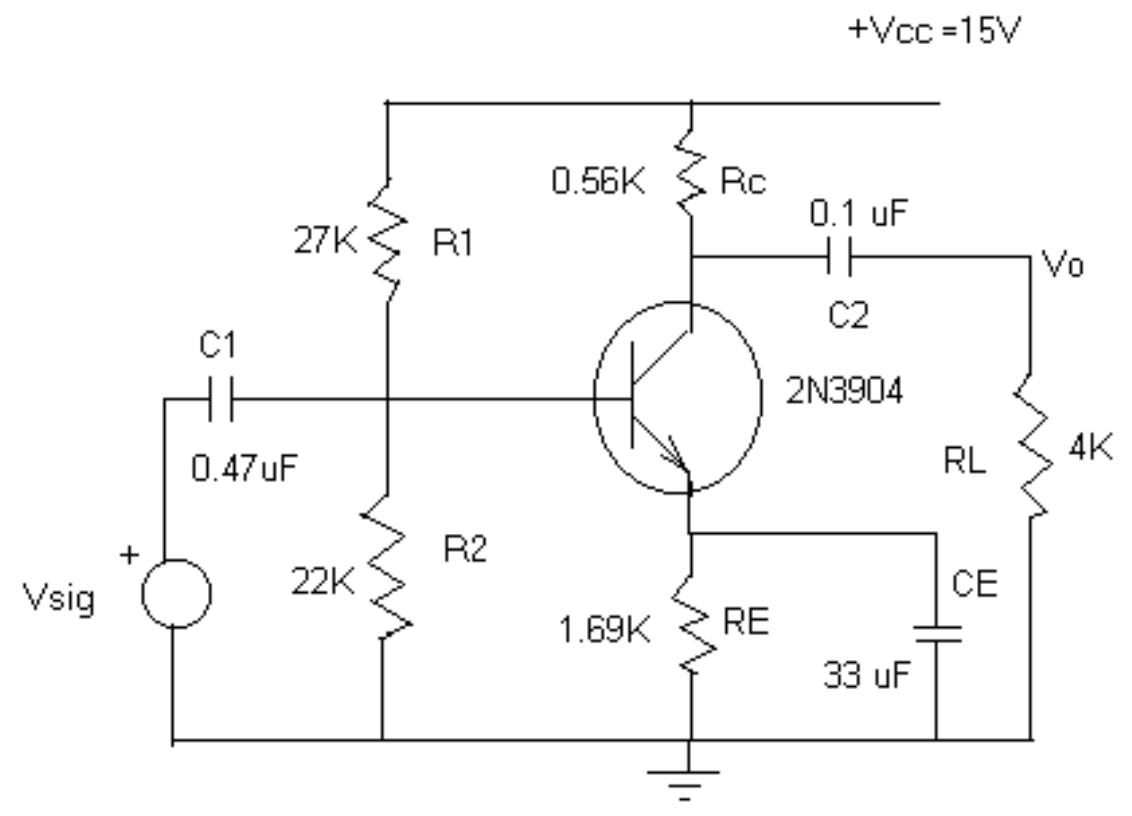

Figure 11. Final design circuit

8-level deep, and the whole tree is very large. After pruning the tree, a large number of possible circuit configurations are obtained. The "best" circuit structure or concept is selected for the system design. Table 2 shows the test results of the actual amplifier circuit. The results are all within the tolerances of the design.

\section{Conclusion}

The Modified Decision Tree Approach in Electrical Engineering design is presented. The method is very useful in generating alternative courses of actions, and in putting the design in proper context, through the discovery of "hidden" design criteria. Using this method, teaching engineering design can be systematized and facilitated. The method can facilitate the creation of documents that will show the design experience of students, for ABET outcome assessment. Finally, the author believes that once students are exposed to this method, it will profoundly change the way they look at engineering design.

\section{Acknowledgments}

The author would like to thank the reviewer, Col. Steve Klegka, for his insightful comments and helpful suggestions for improving the paper. 
York, 1978, pp. 173-182.

2) Hurley, R. B., Decision Tables in Software Engineering, Van Nostrand Reinhold Data Co., New York, 1983.

3) Ertas, A. \& Jones, J., The Engineering Design Process, 2 nd ed., John Wiley \& Sons, New York, 1993, pp. 75-79.

4) Wilcox, A.D., Engineering Design Project Guidelines,, Prentice-Hall, Englewood Cliffs, New Jersey, 1987.

5) Boylestad, R and Nashelsky, L., Electronics Devices, 7th ed., Prentice-Hall, Englewood Cliffs, New Jew Jersey, 1999, Chapter 11.

6) Gottfried, B. S., \& Weisman, J., Introduction to Optimization Theory, Prentice-Hall, Englewood Cliffs, New Jersey, 1973.

DOMINGO L. UY

Domingo Uy is currently an Assistant Professor of Electronics Engineering Technology at Fort Valley State University in Fort Valley, Georgia. Prior to joining FVSU, he had taught the capstone Electrical Engineering Design courses and other major courses in Electrical Engineering, for nine years at Hampton University Electrical Engineering Department. Dr. Uy is a registered Professional Engineer (Electrical) in the State of Ohio and the Commonwealth of Virginia. He received his B.S.E.E. degree from the University of Santo Tomas, M.S.E.E. degree from the University of the Philippines, and Ph.D.E.S. (Systems Theory \& Engineering (Control)) degree in 1987 from The University of Toledo in Toledo, Ohio. Dr. Uy is a member of ASEE and IEEE. 